\title{
Envelhecimento, autocuidado e memória: intervenção como estratégia de prevenção
}

\author{
Aging, self-care and memory: intervention as prevention \\ strategy \\ Envejecimiento, el autocuidado y la memoria: la \\ intervención como estrategia de prevención \\ Eunice Maria Godinho Morando
Juliana Campos Schmitt
Maria Elisa Caputo Ferreira
}

RESUMO: Esta revisão, nas bases Pepsic, Lilacs e SciELO, analisa a relação entre envelhecimento, autocuidado e memória; apresenta as pesquisas sobre autocuidado no Brasil e os profissionais que trabalham com este conceito. Os idosos relatam dificuldade de guardar e recuperar informações, o que interfere no autocuidado. Este exige participação ativa e responsável, influi na autonomia e independência, e favorece a integração do idoso na família e na comunidade. A intervenção é eficaz e benéfica para desenvolver o autocuidado em idosos.

Palavras-chave: Envelhecimento; Autocuidado; Memória.

ABSTRACT: This review, in Pepsic, Lilacs and SciELO databases, analyzes the relationship between aging, self-care, and memory; presents self-care research in Brazil and the workers who work with this concept. The elderly report difficulty in storing and retrieving information, which interferes with self-care. This requires active and responsible participation, influences autonomy, independence, and favors the integration of the elderly into the family and community. Intervention is effective and beneficial in developing self-care in the elderly.

Keywords: Aging; Self-care; Memory. 
RESUMEN: Esta revisión en las bases Pepsic, Lilacs, SciELO analiza la relación entre envejecimiento, autocuidado, memoria; presenta la investigación sobre el autocuidado en Brasil y los trabajadores que trabajan con este concepto. Los ancianos reportan dificultades para almacenar y recuperar información, lo cual interfiere con el autocuidado. Esto requiere participación activa y responsable, influye en la autonomía y independencia y favorece la integración de los ancianos en la familia y comunidad. La intervención es eficaz y beneficiosa para desarrollar el autocuidado en los ancianos.

Palabras clave: Envejecimiento; Autocuidado; Memoria.

\section{Introdução}

Leventhal, Weinman, Leventhal e Phillips (2008), em revisão da pesquisa comportamental com embasamento teórico utilizado no estudo da conduta em relação às doenças crônicas, concluíram que as intervenções comportamentais são eficazes para melhorar os resultados de saúde, e que há possiblidade de desenvolvimento de intervenções cognitivo-comportamentais eficazes e eficientes, baseadas em modelos conceituais oriundos dos processos de resolução de problemas implícitos no autocuidado. Eles propõem que a integração dos conceitos em autogestão pode gerar intervenções comportamentais adaptadas que irão melhorar a qualidade dos cuidados.

Os autores argumentam que, embora não se possa negar a carga genética do indivíduo, os comportamentos sejam eles saudáveis ou de risco, a aderência ao tratamento, e o estilo de vida influenciam na prevenção e no controle de doenças crônicas. Isso significa que a competência cognitiva para a saúde parece ser mediada pelo comportamento. Logo, o modo mais eficaz de afetar os resultados de saúde seria por meio de ações que possam influenciar estes comportamentos, tais como programas de intervenção.

Existem dois conceitos do sistema do self que usualmente são empregados na pesquisa do comportamento em saúde: a auto-eficácia (Bandura, 1989, 2004) e o controle interno (Rodin, \& Langer, 1996). A auto-eficácia se traduz na percepção de que a pessoa é capaz de agir de maneira eficaz para a preservação da própria saúde, e o controle interno pode ser entendido como a percepção de controle pessoal e a responsabilização pelas próprias maneiras de atuar no cotidiano. Ambos são conceitos-crença dinâmicos, plasmados pelo comportamento, que se influenciam mutuamente. 
No mais das vezes, os estudos se dirigem para a demonstração da maneira pela qual a eficácia afeta o comportamento, como, por exemplo: o uso de medicação (Barclay, et al., 2007), alimentação saudável (Shields, \& Brawley, 2006) e exercício (Schwarzer, 2005).

No entanto, é necessário que se verifique também o inverso, ou seja, como o comportamento afeta a eficácia, como o fizeram Maibach e Murphy (1995). Isso porque experienciar afeto positivo, e contar com apoio social, aumenta a auto-eficácia, como demonstraram Dechamps, Lafont, e Bourdel-Marchasson (2007), em relação ao exercício físico. Estas vivências engendram o "significado da ação na memória cognitiva e experiencial; o feedback positivo baseado na experiência transmite o valor do comportamento ao sistema cognitivo" (Leventhal, et al., 2008, p. 490) ${ }^{1}$.

Os programas de intervenção exercitam comportamentos mais adequados para arrefecer ou eliminar um problema de saúde, ou os comportamentos mais favoráveis ao atendimento das necessidades para a manutenção da saúde, do bem-estar ou da qualidade de vida dos indivíduos. O objetivo geral de um programa de intervenção é mobilizar as pessoas para que possam promover mudança de comportamento, através do apoio fornecido pelos profissionais de saúde. Os objetivos específicos se referem às mudanças em determinados comportamentos, que são necessárias para se atingir uma melhora na saúde, bem-estar e qualidade de vida, utilizando-se metodologia precisa, além de ações e técnicas planejadas.

\section{O autocuidado}

Podem-se detectar quatro palavras envolvidas na compreensão do que seja o autocuidado: i) auto, elemento de composição, do grego autós, que exprime a ideia de "por si próprio", "de si mesmo" (Michaelis, 2000, p. 263); ii) cuidado, substantivo masculino com os sentidos de desvelo, diligência, solicitude, atenção ou precaução, vigilância (Michaelis, 2000 p. 622); iii) autonomia, substantivo feminino do grego autonomía, que significa liberdade moral ou intelectual (Michaelis, 2000, p. 266); iv) ética, substantivo feminino, do grego ethiké, parte da filosofia que estuda os valores morais e os princípios ideais da conduta humana (Michaelis, 2000, p. 908).

\footnotetext{
${ }^{1}$ Tradução da autora principal.
} 
Assim, pode-se entender autocuidado como a atenção e a ação que o indivíduo efetua sobre si próprio para desenvolver e conservar uma boa qualidade de vida, agindo de modo livre, autônomo e responsável na escolha dos instrumentos para atingir esse objetivo.

Autocuidado implica observar-se e captar o estado de suas condições físicas, mentais e emocionais, perceber as causas de seus desconfortos, com o intuito de atuar sobre elas de um modo que seja saudável e benéfico. Autocuidado é o processo pelo qual uma pessoa sem formação em saúde age em próprio proveito para promover a saúde pessoal, fazer a prevenção e a detecção de doenças e desenvolver o tratamento destas com base nos recursos primários do sistema de saúde (Levin, Katz, \& Holst, 1976).

Para ter boa saúde, é necessário refletir qual é o estilo de vida que se tem, e criar e sustentar hábitos saudáveis, os quais estão relacionados com a alimentação, atividade física, lazer, além de outras mudanças que venham a promover o bem-estar e a evitar o surgimento ou complicações de doenças. Em suma: autocuidado é olhar para si mesmo, observar-se, eleger ações, e adotar meios de cuidar da própria saúde. O princípio fundamental do autocuidado é o indivíduo como centro de mudança da própria vida e saúde, pois é quem mais e melhor conhece sua situação e sabe do que precisa para se sentir bem. $\mathrm{O}$ autocuidado implica na prática de atividades realizadas por uma pessoa em proveito pessoal, visando à conservação da própria saúde e bem-estar (Geoge, 1993).

Motivação e informação são os pilares do autocuidado. As informações e as orientações, usualmente, são feitas pelas equipes de saúde, mas também podem ser realizadas pela família e pelos amigos, podendo ainda contar com a ajuda de espaços diversos, tais como: igreja, escola, associações de bairro, associações profissionais e internet. Os profissionais de saúde, incluindo os psicólogos, têm o dever de orientar, cooperar e assistir as pessoas no percurso do desenvolvimento do autocuidado.

Horta (1979) afirma que é função dos profissionais de enfermagem auxiliar os indivíduos, assistindo-os nas suas necessidades elementares, por meio do ensino do autocuidado para preservar e promover a saúde, capacitando-os tanto a serem ativos e comprometidos na identificação de suas dificuldades para realizar os próprios cuidados, quanto no encontro de solução para elas.

Bertoletti, e Junges (2014) consideram que o psicólogo precisa compreender o processo de declínio advindo do envelhecimento como consequência natural do curso da vida e estar sensível às realidades peculiares dos idosos. 
Para que possa lhes oferecer uma efetiva ajuda, este profissional deve fazer uma análise crítica do autocuidado que os idosos dispensam a si mesmos para, assim, poderem alcançar a compreensão das experiências de vida que tiveram, obtendo como resultado o atuar a favor da obtenção da qualidade de vida e do bem-estar subjetivo.

Segundo Derntl, e Watanabe (2004), a concepção de autocuidado não deve estar restrita às ações que busquem a modificação de estilos de vida nocivos à saúde, mas entendem que os valores e determinantes sócio-econômico-culturais devem estar incluídos na abordagem desta questão, para que se tenha uma correta compreensão do que ela seja e do modo pelo qual deve ser tratada nos programas de intervenção.

A avaliação da percepção e do entendimento dos idosos a respeito do autocuidado deve ser feita, estabelecendo-se a relação entre as suas modificações corporais e sua capacidade de atuar em diferentes situações com os sentimentos e as reações, por vezes negativas, dos familiares e demais pessoas que são importantes para eles.

Desenvolver o autocuidado é um processo a ser compreendido e facilitado pelos profissionais da saúde, porque, para que se venha a obter êxito, não se pode olvidar o atendimento das necessidades de auto-imagem e auto-estima que estão estreitamente ligadas à manutenção do estilo de vida dos idosos, independentemente da diminuição das suas capacidades (Duarte, 1998).

Bertoletti, e Junges (2014) compreendem o autocuidado como um elemento constitutivo da subjetividade humana. Em uma pesquisa sobre a percepção de idosas octogenárias sobre o autocuidado, os autores concluíram que envelhecimento, a qualidade de vida e a valorização social estão essencialmente associadas ao autocuidado.

\section{A relação entre memória e autocuidado em idosos}

A questão da memória é um problema de saúde que interfere no autocuidado, uma vez que as queixas reportadas pelos idosos referem-se à dificuldade de guardar informações e de recuperá-las quando necessário. Entre outras, as queixas mais frequentes são: não se recordar de nomes de pessoas conhecidas ou de compromissos importantes; não se lembrar de tomar medicação ou ficar na dúvida se a tomou ou não; não se lembrar em que lugar guardaram o seu dinheiro ou deixaram objetos pessoais, como os óculos; esquecer o fogo aceso, o ferro ligado ou a torneira aberta. 
Estes esquecimentos prejudicam o desempenho no trabalho, a atuação na vida social, familiar e afetiva, e a participação civil e pública, influem em sua segurança e na dos familiares, afetando negativamente seu cotidiano, podendo causar prejuízo pessoal, ocupacional e social, e levar ao auto-abandono, à perda da auto-estima e ao isolamento da sociedade, e até mesmo do ambiente familiar. Assim, a capacidade cognitiva é um importante determinador da qualidade de vida na velhice (Ribeiro, \& Yassuda, 2007).

Dias, Andrade, Duarte, Santos, e Lebrão (2015) estabeleceram relação entre o declínio cognitivo e a realização de atividades avançadas da vida diária, constatando que a média de desempenho destas atividades foi significativamente maior nos idosos sem declínio cognitivo.

As dimensões subjetivas, que conformam o conceito de qualidade de vida para uma pessoa, estão intimamente relacionadas com o desempenho das funções cognitivas. Sendo o desempenho um dos agentes causadores da adequação do comportamento e da resolução de situações e problemas da vida diária, seu declínio altera tanto a qualidade de vida quanto a percepção subjetiva da mesma (Yassuda, \& Abreu, 2007).

A preocupação com a manutenção da saúde do idoso, de sua independência e autonomia é importante e relevante em vários aspectos: humano, social e econômico e demonstra um tipo de evolução cultural, uma vez que busca impedir tanto a desumanização e a estigmatização do idoso (Goffman, 1982), quanto à desumanização e o preconceito contra ele (Beauvoir, 1970).

Neste contexto, deve-se ter em mente que as queixas em relação a seu desempenho mnemônico são reais e que precisam ser acolhidas com respeito, compreendendo que sejam elas decorrentes de um processo natural ou de um declínio cognitivo fisiológico sugestivo de demência, e necessitam de intervenção com vistas à redução ou eliminação deste transtorno.

Com o envelhecimento, pode haver um continuum de perdas, conformando um espectro que vai desde as deficiências leves até atingir os quadros mais graves que incluem as demências. Neste último caso, as perdas são importantes e passam a intervir na execução das atividades da vida diária, culminado na perda da autonomia e da independência (Clemente, \& Ribeiro-Filho, 2008). 
Ogden (2004) apresenta como um dos objetivos da psicologia da saúde: compreender, explicar, desenvolver e testar teorias. Assim, um programa de intervenção do treinamento da memória pode ser uma estratégia eficaz e efetiva para a melhoria da capacidade mnemônica, produzindo efeitos na qualidade de vida de idosos saudáveis, e consistindo em uma possível solução para os reclames deste tipo de perda, bem como uma alternativa eficaz e efetiva de atendimento à população idosa.

A manutenção da autonomia e da independência do idoso, por um mais dilatado período de tempo, aumenta a possibilidade de melhoria no autocuidado e consequente preservação da saúde. Esta cadeia de eventos afeta de modo positivo o sistema de saúde pela prevenção da doença na população idosa e consequente redução de seus custos. A manutenção da capacidade funcional é importante para que não haja uma sobrecarga no sistema de saúde.

Kalache (1997) argumenta que, além da mudança demográfica, a urbanização, a industrialização, e o ingresso da mulher no mercado de trabalho provocaram alterações nos valores sociais tradicionais, acarretando transformações na dinâmica e na composição familiar. Do aumento da população idosa, decorrem maiores gastos estatais para a manutenção da parte economicamente improdutiva. A confluência de todos estes fatores se reflete nos cuidados com a população idosa, que, tradicionalmente, recebia atenção e cuidados primordialmente da família e vem sendo, gradual e crescentemente, substituída pela intervenção do Estado e de outras formas de atendimento institucional.

Neri (2008) e Papalia, Olds, e Feldman (2006) relatam perdas advindas do processo de envelhecimento ligadas às capacidades cognitivas, como o processamento da informação, memória e aprendizagem, que entram em declínio por causa das alterações neurológicas e sensoriais correlatas a este processo. $\mathrm{O}$ decaimento da rapidez perceptiva parece se associar à perda da memória, o que atinge a eficiência do processamento das informações (Papalia, Olds, e Feldman, 2006).

De acordo com Berger (1995), a habilidade de recordar em um período breve de tempo decai com a idade, mormente a memória de curto prazo, que é acompanhada de deterioração semelhante no desempenho das tarefas que demandam velocidade e boa memória de curto prazo (lembrança de informação por poucos segundos ou horas, segundo a Biblioteca Virtual de Saúde, BVS). 
Por outro lado, mantém-se a memória de longo prazo e as estratégias usuais de resolução de problemas, o vocabulário, a informação acumulada e a aprendizagem realizada, o que é confirmado por Papalia, Olds, e Feldman (2006). Estes últimos ressaltam que os idosos requerem um tempo maior para a resolução de tarefas, que o desempenho cognitivo revela uma plasticidade importante, a qual pode ser favorecida com algum treino, e ainda uma relação positiva entre idosos saudáveis e melhor funcionamento intelectual. Os idosos considerados normais são capazes de manter um bom desempenho na atenção seletiva, ou seja, quando a exigência de concentração se restringe a um único objeto ou evento.

No entanto, nas situações em que há uma exigência de divisão da atenção, por exemplo, na realização de tarefas simultâneas, seu desempenho é pior do que o do adulto jovem (Camargo, 2006).

Melhorar o desempenho das funções cognitivas em idosos traz consequências importantes para sua qualidade de vida. A literatura sugere que o treinamento pode aprimorar as funções cognitivas em idosos saudáveis (Lira, Rugene, \& Mello, 2011). Para Irigaray (2009), há melhoria no desempenho cognitivo, na qualidade de vida e no bem-estar psicológico de idosos saudáveis com o treino cognitivo.

Investigar as condições que interferem na qualidade de vida dos idosos, com o fim de serem criadas alternativas de intervenção, e de se proporem ações e políticas para a área de saúde, que possam dar uma resposta efetiva às necessidades e aos reclames da população idosa (Silva, 2009), isso tudo possibilita o desenvolvimento do autocuidado.

A necessidade de mais estudos na área do envelhecimento, que busquem verificar a relação entre funcionamento cognitivo e o declínio da memória, é sustentada por vários autores. Entre eles: Cavallini, et al. (2015); Sandberg, Rönnlund, Nyberg, e Neely (2014); Rojas, e Villanea (2014); Silva, et al. (2014); Fabrício, et al. (2012); Chariglione, e Janczura (2013); Maseda, Millán-Calenti, Lorenzo-López, e Núñez-Naveira (2013).

Os efeitos benéficos do treinamento das funções cognitivas e de alguns tipos de memória em idosos são defendidos por estes mesmos autores, cujas pesquisas trabalharam, respectivamente, com: - um treinamento cognitivo de auto-ajuda que incluía a prática de tarefas de aprendizagem, e tarefas com instruções para transferência; - a possibilidade de fortalecimento do executivo, com um programa centrado em várias funções executivas; - a verificação de associação entre a eficácia do treino e as características da intervenção (pequenos grupos, uso de tarefas ecológicas, pareamento por nível de escolaridade); - o funcionamento cognitivo de idosos ativos em San Jose, na Costa Rica; 
- as relações entre queixas de memória, sintomas depressivos e desempenho cognitivo; - a verificação de associação entre a eficácia do treino às características da intervenção; - a influência de diferentes treinos cognitivos de memória em idosos institucionalizados e em medidas neuropsicológicas e de humor; - a correlação entre os fatores sociodemográficos (idade, sexo e educação) e a pontuação total e os subscores da tela de 7 minutos.

Pode-se concluir, levando-se em conta todos estes autores e suas pesquisas, que há influência positiva dos treinamentos cognitivos em idosos, que eles são eficazes para promover a preservação do funcionamento cognitivo e cumprem o objetivo essencial de adiamento ou prevenção do declínio cognitivo.

Dias, et al. (2015) estabeleceram relação entre o declínio cognitivo e a realização de atividades avançadas da vida diária, constatando que a média de desempenho destas atividades foi significativamente maior nos idosos sem declínio cognitivo.

A preservação da memória está diretamente ligada à possibilidade do autocuidado em idosos. Verdan, et al. (2014), em estudo sobre a mortalidade em pacientes diagnosticados com demência, demonstram que, independentemente da idade, gênero, e escolaridade, a mortalidade decresce na proporção do aumento da funcionalidade e guarda relação com o estado cognitivo e basal. Já Sato, Batista, e Almeida (2014) relatam que as dificuldades cognitivas são compensadas pela aprendizagem de estratégias em treinamento cognitivo e que a estimulação da memória contribui para a melhor realização das atividades cotidianas.

\section{A intervenção como estratégia de desenvolvimento do autocuidado}

Intervenção é um substantivo feminino que significa ato ou efeito de intervir, e indica uma intercessão ou mediação em alguma situação adversa.

$\mathrm{Na}$ medicina, uma intervenção é uma operação ou procedimento cirúrgico, realizado com o fito de tratar alguma doença de um paciente (Michaelis, 2000, p. 1170).

A palavra em inglês para intervenção é intervention, que também tem o sentido de intercessão e mediação, e se apresenta na vida das pessoas em geral pela manifestação de apoio de amigos e/ou familiares, para uma pessoa com problemas de comportamento ou de drogadição. Há uma intervention, quando amigos ou familiares identificam um problema, e se dispõem a prestar uma ajuda, para promover a eliminação ou minimização do problema. 
Os programas de intervenção objetivam exatamente isto: a identificação correta de um problema, o fornecimento de informação a respeito dele, e o uso de metodologia, técnicas, e ações que permitam trabalhar na erradicação ou diminuição do problema, ou de seus efeitos na vida dos indivíduos.

\section{Método}

Para verificar o que tem sido feito em termos de pesquisa a respeito de programas de intervenção e autocuidado com idosos, foi realizada uma pesquisa em novembro de 2016, em três bases de dados: Pepsic, Lilacs e SciELO, sem determinação do período de tempo da produção científica. Em uma primeira busca, foram usados os descritores "autocuidado", “idoso" e "intervenção", encontrando-se na Pepsic 1 artigo; na Scielo, 3 e na Lilacs, 45.

Do total de 49 artigos encontrados, 33 eram da área de Enfermagem; 8 da Psicologia, havendo 5 repetições; 3 da interseção da Educação Física com a Geriatria; 2 da Psiquiatria; 2 da Fisioterapia e 1 da Terapia Ocupacional. Os números demonstram que a Enfermagem é a área que se sobressai na produção científica do tema e as demais áreas pouco tratam dele.

Como o número de artigos que abordam o autocuidado em uma perspectiva psicológica foi pequeno, e objetivando ter uma visão mais específica da produção científica do tema em estudo, procedeu-se à realização de novas buscas, combinando-se os dois descritores anteriormente usados com os descritores "treino cognitivo-comportamental", "treino cognitivo", "treino comportamental", treinamento cognitivo-comportamental", "treinamento cognitivo", "treinamento comportamental”, "intervenção cognitivocomportamental”, “intervenção cognitiva”, “intervenção comportamental”. Com a adição destes descritores, não foi encontrado artigo algum na Scielo. Na Lilacs, foram encontrados 2 artigos com a adição do descritor "intervenção cognitiva", o que não trouxe contribuição à busca anterior por serem repetidos; com os demais descritores não foi encontrado nenhum artigo. Na Pepsic, foi encontrado um único artigo com a adição do descritor "treinamento cognitivo"; com os demais descritores, não houve registro algum. Assim, o total de artigos encontrado foi 52 . 
Foram excluídos 7 artigos por serem repetidos e 30 pelas seguintes razões: não ser programa de intervenção (2); ser programa de intervenção, mas não tratar do autocuidado (13); não ser programa de intervenção e nem tratar de autocuidado (14); artigo de revisão (1). Deste modo, restaram 15 artigos que são analisados a seguir.

\section{Resultados}

Dentre as quinze pesquisas analisadas, quatorze concluíram que a estratégia de intervenção é eficaz e benéfica, apresentando resolutividade do problema de saúde, e melhoria no empoderamento do paciente e no autocuidado. O estudo de Nicolato, Couto e Castro (2016) destacou que a capacidade de realizar o autocuidado é influenciada pelos fatores: idade, gênero, experiências de vida, escolaridade, valores, crenças, aspectos culturais do sujeito e dos recursos que estão disponíveis para ele. Bringuente, Castro, Jesus e Luciano (1997) também consideraram que o autocuidado deve ser ministrado, tendo em consideração a condição social, histórica e cultural dos indivíduos, enquanto Borges e Seidl (2013, 2014) ressaltaram os fatores pessoais, demográficos e culturais.

\section{Discussão}

Nicolato, et al. (2016) utilizaram o modo de intervenção apoio-educação, proposto por Dorothea Orem, que emprega ações de orientação e ensino de autocuidado terapêutico, para analisar a capacidade de autocuidado de idosos atendidos pela consulta de enfermagem em um ambulatório multiprofissional de atenção à saúde do idoso. As autoras concluíram que o idoso manifesta comportamento de procura de atendimento na atenção secundária à saúde, objetivando aprender a desenvolver o autocuidado geral e terapêutico.

Borges e Seidl (2013, 2014) pesquisaram os efeitos de uma intervenção psicoeducativa para fortalecer o autocuidado em homens idosos e concluíram que existe utilidade da intervenção na compreensão das vantagens do desenvolvimento de ações preventivas, ou de controle de doenças, no reforço de práticas saudáveis, e na adoção de mudanças necessárias e/ou almejadas. 
Ressaltam que, na presença de barreiras psicológicas à mudança, faz-se necessário associar as intervenções grupais, intervenções individuais, que possam ajudar os idosos a rever suas crenças, melhor gerenciar as respostas emocionais, e aprender novas respostas para situações produtoras de estresse. Constataram ainda que o contato educativo com profissionais, e seus pares, é benéfico, ao permitir a troca de experiências e conhecimentos, fomentando o autocuidado e influindo na mudança de comportamentos nocivos à saúde. Alertam para que não se incorra no erro de considerar que os homens constituem um corpo homogêneo com valores e comportamentos comuns e generalizáveis, porque isto pode comprometer o trabalho de promoção da saúde. As práticas de saúde voltadas para o sexo masculino devem ser diversas, uma vez que se vinculam aos fatores demográficos, pessoais, e culturais (Borges, \& Seidl, 2013, 2014; Costa, 2014).

Costa (2014) relata que a ação educativa com intervenção em um grupo para pacientes de diabetes propiciou o compartilhamento de experiências, a discussão das barreiras e as alternativas para ultrapassá-las no esforço para se colocar em prática o propósito de atingir o autocuidado, e resultou em melhoria no empoderamento e no autocuidado, tornando-os mais aptos a se envolver e a cooperar na gestão da própria saúde.

Maia, Reis, e Torres (2016) afirmam que há relação entre a duração de um programa educativo para portadores de diabetes mellitus e a aquisição do conhecimento e habilidades de autocuidado. A conclusão é que a melhoria ocorre com intervenção educacional de oito horas ou mais e que, para haver eficácia da promoção de competências relacionadas ao conhecimento e autocuidado em diabetes mellitus, a duração do programa educacional é fator relevante.

Batista, Becker, Zanetti, e Teixeira (2013), ao analisarem a habilidade de pessoas com diabetes mellitus na auto-aplicação de insulina, constataram erros ou omissões na realização das etapas deste processo. Após intervenção educativa em grupo, para a correta realização desse procedimento, houve diminuição dos erros e omissões, evidenciando-se que a atividade contribuiu para o autocuidado no tratamento insulinoterápico.

Pereira, Costa, Sousa, Jardim, e Zanini (2012) pesquisaram o efeito da ação educativa no conhecimento da doença em pessoas com diabetes tipo 2. Houve aumento significativo do conhecimento da doença em todos os aspectos nas pessoas que participaram das atividades educativas em grupo, concluindo pela eficácia da ação educativa. 
Torres, Franco, Stradioto, Hortale, e Schall (2009) também pesquisaram este mesmo tema, comparando a efetividade de um programa educativo em diabetes aplicado individualmente e em grupo, e constataram semelhança nos resultados dos testes de atitudes, mudanças de comportamento e qualidade de vida. No entanto, a redução dos níveis de hemoglobina glicosilada foi significativa apenas nos pacientes integrantes da intervenção feita em grupo. Concluíram que ambas as formas de trabalho educativo surtem resultado, mas que o trabalho em grupo é mais eficiente no controle glicêmico.

Silva (2012) verificou haver benefício na aplicação da terapia comportamental em mulheres com incontinência urinária. A autora ressalta que a informação e a orientação recebidas de um profissional é uma estratégia de promoção da saúde, que produz o hábito do autocuidado, o qual se reflete em melhoria nos aspectos emocional, social, e econômico do indivíduo incontinente.

Rodríguez-Gázquez, Arredondo-Holguín, e Herrera-Cortés (2012) avaliaram a efetividade de um programa educativo de enfermagem, na melhoria dos comportamentos de autocuidado em pacientes com insuficiência cardíaca e, com base nos resultados, concluíram que este tipo de intervenção tem efeito benéfico nos comportamentos de autocuidado das pessoas com esta questão de saúde.

Domingues, Clausell, Aliti, Dominguez, e Rabelo (2011) também pesquisaram pacientes com insuficiência cardíaca, e verificaram que a intervenção educativa de enfermagem intra-hospitalar trouxe benefícios para eles, no que diz respeito ao conhecimento da doença e autocuidado, após a alta hospitalar.

Morais (2009) relata que a intervenção em grupo com idosos que passaram por cirurgia de próstata, objetivando a conscientização do autocuidado obteve o resultado de desenvolvimento da responsabilização pela própria saúde. Realça a importância da intervenção do psicólogo como agente facilitador do processo grupal no atendimento aos idosos, e a relevante contribuição deste tipo de trabalho na aquisição e/ou manutenção de uma velhice saudável.

Silva (2000) também realizou uma intervenção com indivíduos submetidos à cirurgia de próstata, oferecendo-lhes, na consulta de enfermagem, orientação para o autocuidado, segundo a teoria de Orem. Concluiu pelo benefício da intervenção como terapêutica para recuperação da doença e no enfrentamento de seus efeitos. 
Bringuente, et al. (1997) estudaram os fatores de risco para a pessoa com dor na coluna, em uma intervençäo realizada por meio de um programa de educação à saúde, com base no ensino do autocuidado, pressupostos filosóficos humanistas-existenciais, e abordagens equalizadoras do estresse. Concluíram que o programa produziu melhora na frequência e intensidade dos eventos de dor, com ganhos no conhecimento do fenômeno dor na coluna, no autoconhecimento e na aprendizagem do cuidado do próprio corpo e da própria saúde. Entenderam que o estilo de vida é um fator de risco passível de modificação pelo ensino do autocuidado, o qual deve ser ministrado, considerando-se o potencial das pessoas e sua condição social, histórica, e cultural.

Santos (1989) elaborou uma proposta de trabalho para o ensino do autocuidado em pessoas com dores crônicas, baseada em medidas terapêuticas alternativas, sob o enfoque holístico. Concluiu que o trabalho de enfermagem tem grande contribuição na resolutividade da dor crônica pelo ensino do enfrentamento à dor e à equalização do estresse, propiciando ganhos na qualidade de vida. Ressaltou o papel pedagógico do profissional da enfermagem.

\section{Considerações Finais}

A pesquisa a respeito de programas de intervenção e autocuidado com idosos foi realizada em novembro de 2016 em três bases de dados: Pepsic, Lilacs e SciELO, sem determinação do período de tempo da produção científica.

De acordo com ela, as especialidades da área da saúde que empregam e pesquisam o autocuidado no Brasil são: enfermagem (33 artigos), psicologia (3), psiquiatria (2), geriatria/educação física (2), fisioterapia (2) e terapia ocupacional (1). Pelo número de artigos encontrados em cada especialidade, pode-se perceber que a enfermagem é a que se sobressai na prática do autocuidado e na produção científica, sendo que as demais áreas pouco tratam deste tema.

Isso pode ser explicado pelo fato de a enfermagem contar com uma teoria do autocuidado bastante desenvolvida e consolidada: a de Dorothea Orem. Esta teoria concebe o autocuidado como a prática de atividades para a manutenção da vida, da saúde e do bemestar. Considera que, se efetivamente realizado, o autocuidado colabora para a manutenção do bom funcionamento orgânico e físico do organismo humano. 
Afirma que o autocuidado está sujeito às condicionalidades: gênero, idade, estado de saúde, condição sociocultural, sistema familiar, padrão econômico, fatores ambientais, adequação e disponibilidade de recursos, modalidade de diagnóstico e tratamento.

O envelhecimento da população brasileira e mundial tem sido objeto de preocupação dos governantes e dos profissionais de saúde, pois o aumento da longevidade tem sido acompanhado por problemas de saúde de maior duração, o que traz ônus tanto para o indivíduo quanto para a sociedade.

As doenças crônicas e as complicações dela decorrentes podem estar associadas aos comportamentos dos indivíduos, mas também aos dos profissionais da saúde. A assistência e os encaminhamentos geralmente são emergenciais, sendo dispensada pouca atenção à prevenção e ao controle dos problemas de saúde em todas as faixas etárias da população e, particularmente, quando se trata dos idosos.

Além da óbvia responsabilidade governamental pelo sistema nacional de saúde nas três esferas de poder, o desafio a ser enfrentado pelos profissionais de saúde é saber como podem colaborar na prevenção dos problemas de saúde, e quais práticas podem fazer face às novas demandas diante deste quadro do envelhecimento populacional. O Ministério da Saúde estabelece que as ações de promoção da saúde do idoso devem incluir o autocuidado, além do cuidado familiar e institucional.

O autocuidado como estratégia de atenção à saúde tem como objetivo manter os idosos em seu ambiente familiar e social, guardando o maior grau de autonomia e independência pelo maior período de tempo possível.

O autocuidado tem como base a compreensão de que o homem é dotado da capacidade de reflexão, capacidade esta que aplica sobre si e sobre o mundo, simbolizando suas experiências e desenvolvendo motivação para cuidar de si e cuidar do outro.

O autocuidado implica a prática de atividades realizadas e mantidas ao longo do tempo por uma pessoa em proveito pessoal, com o intuito de conservação da própria saúde e de seu bem-estar.

Para que o autocuidado seja seguro e eficaz, e não somente focado no aspecto econômico, é mister que haja o desenvolvimento da competência não somente do indivíduo, mas também dos profissionais de saúde, de modo que possam prestar informações e orientações corretas e técnicas, auxiliando a população no desenvolvimento desta competência. 
O desenvolvimento da competência para o autocuidado é especialmente importante quando se trata dos idosos, porque as necessidades de saúde sofrem transformações durante o processo de envelhecimento. Por isso, o trabalho de intervenção é particularmente importante para a população idosa.

O fato de o autocuidado exigir participação ativa e responsável por parte do indivíduo colabora para a manutenção da sua autonomia e independência, as quais, por sua vez, favorecem sua integração no meio familiar e comunitário.

O autocuidado, de algum modo, reconhece as habilidades e o potencial dos idosos para novas aprendizagens e os reconhece como sujeitos capazes de agir e participar das decisões sobre a própria vida, afastando a concepção de pessoa passiva e dependente.

A psicologia, como um domínio específico na busca de solução para os problemas dos indivíduos, tem uma importante colaboração a prestar, auxiliando os idosos a se manterem ativos e a preservarem sua independência e autonomia, dando-lhes condições de melhor compreenderem os aspectos biopsicossociais incluídos no processo do envelhecimento.

Os trabalhos de intervenção para a promoção do autocuidado fortalecem a manutenção de estilos de vida saudáveis, influenciando a mudança de comportamentos nocivos à saúde; fomentam o autocuidado, permitem a troca de experiências e conhecimentos e colaboram para que o envelhecimento seja bem-sucedido.

Os trabalhos de intervenção que exercitam a memória vão propiciar uma melhor qualidade de vida para os idosos, na medida em que retardam o declínio cognitivo e permitem a realização das atividades da vida diária com independência por um tempo mais prolongado.

A atuação do psicólogo como agente facilitador do processo grupal no atendimento aos idosos nos trabalhos de intervenção, seja para a promoção do autocuidado, seja para a estimulação e exercício da memória, é relevante para a conquista e/ou manutenção de uma velhice saudável.

O processo do envelhecimento, por ser uma experiência que transforma os modos de subjetivação, demanda a criação de novos papéis na busca pela autonomia e independência. O psicólogo ao contribuir para que os idosos mantenham estas duas capacidades, além de respeitá-los como seres humanos, ajuda a garantir seus direitos e promove sua inserção social. 


\section{Referências}

Bandura, A. (1989). Self-regulation of motivation and action through internal standards and goal systems. In: Pervin, L. A. (Ed.). Goal Concepts in Personality and Social Psychology, 19-85. Hillsdale: NJ Erlbaum.

Bandura, A. (2004). Health promotion by social cognitive means. Stanford, California: Health Education \& Behavior, 31(2), 143-164. Recuperado em 10 novembro, 2016, https://doi.org/10.1177/1090198104263660.

Barclay, T. R., Hinkin, C. H., Castellon, S. A., Mason, K. I., Reinhard, M. J., Marion, S. D., Levine, A. J., \& Durvasula, R. S. (2007). Age-associated predictors of medication adherence in HIV-positive adults: health beliefs, self-efficacy, and neurocognitive status. Los Angeles, California: Health Psychology, 26(1), 40-49. Recuperado em 2 novembro, 2016, de: http://dx.doi.org/10.1037/0278-6133.26.1.40.

Batista, J. M. F., Becker, T. A. C., Zanetti, M. L., \& Teixeira, C. R. de S. (2013). O ensino em grupo do processo de aplicação de insulina. Revista Eletrônica de Enfermagem, 15(1), 7179. Recuperado em 17 novembro, 2016, de: https://doi.org/10.5216/ree.v15i1.16179.

Beauvoir, S. (1970). A velhice. Maria Helena Franco Martins, Trad. Rio de Janeiro, RJ: Editora Nova Fronteira S. A. (Obra original publicada em 1970).

Berger, L. (1995). Pessoas Idosas. Lisboa, Portugal: Lusodidacta.

Bertoletti, E., \& Junges, J. R. (2014). O autocuidado de idosas octogenárias: desafios à Psicologia. São Paulo, SP: Revista Kairós Gerontologia, 17(3), 285-303. Recuperado em 10 novembro, 2016, de: https://revistas.pucsp.br/index.php/kairos/article/view/23019/16651.

Borges, M., \& Seidl, E. M. F. (2013). Efeitos de intervenção psicoeducativa sobre a utilização de serviços de saúde por homens idosos. Botucatu, SP: Interface Comunicação Saúde Educação, 17(47), 777-788. Recuperado em 12 novembro, 2016, de: http://dx.doi.org/10.1590/S1414-32832013005000024.

Borges, M., \& Seidl, E. M. (2014). Saúde autopercebida e qualidade de vida de homens participantes de intervenção psicoeducativa para idosos. Bragança Paulista, SP: Psico-USF, 19(3), 421-431. Recuperado em 15 novembro 2016, de: http://dx.doi.org/10.1590/141382712014019003005 .

Bringuente, M. E. de O., Castro, I. S. de, Jesus, J. C. G. de, \& Luciano, L. dos S. (1997). Fatores de risco para a coluna: avaliação em consulta de enfermagem. Brasília, DF: Revista Brasileira de Enfermagem, 50(3), 391-406. Recuperado em 15 novembro, 2016, de: http://dx.doi.org/10.1590/S0034-71671997000300008.

Camargo, C. H. P., Gil, G., \& Moreno, M. D. P. Q. (2006). Envelhecimento "normal" e cognição. In: Bottino, C. M. C., Lacks, J., \& Blay, S. L. Demência e transtornos cognitivos em idosos, 13-20. Rio de Janeiro, RJ: Guanabara-Koogan.

Cavallini, E., Bottiroli, S., Capotosto, E., De Beni, R., Pavan, G., Vecchi, T., \& Borella, E. (2015). Self-help memory training for healthy older adults in a residential care center: specific and transfer effects on performance and beliefs. International Journal Geriatric Psychiatry, 30(8), 870-80. Recuperado em 10 novembro, 2016, de: http://onlinelibrary.wiley.com/doi/10.1002/gps.4230/abstract. 
Chariglione, I. P. F., \& Janczura, G. A. (2013). Contribuições de um treino cognitivo para a memória de idosos institucionalizados. Bragança Paulista, SP: Psico-USF, 18(1), 1322. Recuperado em 10 novembro, $2016, \quad$ de: http://www.scielo.br/pdf/pusf/v18n1/v18n1a03.pdf.

Clemente, R. S. G. \& Ribeiro-Filho, S. T. (2008). Comprometimento cognitivo leve: aspectos conceituais, abordagem clínica e diagnóstica. Rio de Janeiro, RJ: Revista Hospital Universitário Pedro Ernesto, 7(1), 68-77. Recuperado em 10 novembro, 2016, de: http://revista.hupe.uerj.br/detalhe_artigo.asp?id=193.

Costa, D. V. de P. (2014). Empoderamento na educação em grupo de diabetes na atenção primária à saúde. Dissertação de mestrado, Universidade Federal de Minas Gerais, PósGraduação em Enfermagem, Belo Horizonte, MG. Recuperada em 10 novembro, 2016, de: http://www.bibliotecadigital.ufmg.br/dspace/handle/1843/ANDO-9M3KPA.

Dechamps. A., Lafont L., \& Bourdel-Marchasson, I. (2007). Effects of Tai Chi exercises on selfefficacy and psychological health. European Review of Aging and Physical Activity, 4(1), 25-32. Recuperado em 20 novembro, 2016, de: https:/link.springer.com/article/10.1007/s11556-007-0015-0.

Derntl, A. M., \& Watanabe H. A. W. Promoção à saúde. (2004). In: Litvoc, J., \& Brito, F. C., (Orgs.). Envelhecimento: Prevenção e promoção à saúde, 37-46. São Paulo, SP: Atheneu.

Dias, E. G., Andrade, F. B. de, Duarte, Y. A. de O., Santos, J. L., \& Lebrão, M. L. (2015). Atividades avançadas de vida diária e incidência de declínio cognitivo em idosos: Estudo SABE. Rio de Janeiro, RJ: Cadernos de Saúde Pública, 31(8), 1623-1635. Recuperado em 18 novembro, 2016, de: http://dx.doi.org/10.1590/0102-311X00125014.

Domingues, F. B., Clausell, N., Aliti, G. B., Dominguez, D. R., \& Rabelo, E. R. (2011). Educação e monitorização por telefone de pacientes com insuficiência cardíaca: ensaio clínico randomizado. Porto Alegre, RS: Arquivos Brasileiros de Cardiologia, 96(3), 233-239. Recuperado em 08 novembro, 2016, de: http://dx.doi.org/10.1590/S0066782 X2011005000014.

Duarte, M. J. R. S. (1998). O envelhecer saudável: autocuidado para a qualidade de vida. Rio de Janeiro, RJ: Revista Enfermagem UERJ, 6, 293-307.

Fabrício, A. T., Lima-Silva, T. B., Kissaki, P. T., Vieira, M. G., Ordonez, T. N., Oliveira, T. B., Aramaki, F. O., Souza, P. F., \& Yassuda, M. S. (2012). Treino cognitivo em adultos maduros e idosos: impacto de estratégias segundo faixas de escolaridade. São Paulo, SP: Psico-USF, 17(1), 85-95. Recuperado em 08 novembro, 2016, de: http://www.redalyc.org/articulo.oa?id=401036088010.

Geoge, J. B. (1993). Teorias de enfermagem. Porto Alegre, RS: Artes Médicas.

Goffman, E. (1982). Estigma - Notas sobre a Manipulação da Identidade Deteriorada. Márcia Bandeira de Mello Leite Nunes, Trad. Rio de Janeiro, RJ: Zahar Editores. (Obra original publicada em 1963).

Horta, W. A. (1979). Processo de enfermagem. São Paulo: EPU/EDUSP.

Irigaray, T. Q. (2009). Efeitos de um treino de atenção, memória e funções executivas na cognição, na qualidade de vida e no bem-estar psicológico de idosos saudáveis. Tese de doutorado, Pontifícia Universidade Católica do Rio Grande do Sul, Programa de PósGraduação em Gerontologia Biomédica, Porto Alegre, RS. Recuperada em 06 novembro, 2016, de: http://repositorio.pucrs.br/dspace/handle/10923/3562. 
Kalache, A., Veras, R., \& Ramos L. R. (1997). O envelhecimento da população mundial. Um desafio novo. São Paulo, SP: Revista de Saúde Pública, 21(3), 200-210. Recuperado em 18 novembro, 2016, de: https://scielosp.org/pdf/rsp/1987.v21n3/200-210/pt.

Leventhal, H., Weinman, J., Leventhal, E. A., \& Phillips L. A. (2008). Health Psychology: The Search for Pathways between Behavior and Health. The Annual Review of Psychology, 59, 477-505. Recuperado em 21 novembro, 2016, de: http://www.annualreviews.org/doi/abs/10.1146/annurev.psych.59.103006.093643.

Levin, L., Katz, A., \& Holtz, E. (1976). Self-care: lay initiatives in healthy. New York: Prodist.

Lira, J. L., Rugene, O. T. \& Mello, P. C. H. (2011). Desempenho de idosos em testes específicos: efeito de Grupo de Estimulação. Rio de Janeiro, RJ: Revista Brasileira de Geriatria e Gerontologia, 14(2), 209-220. Recuperado em 15 novembro, 2016, de: http://dx.doi.org/10.1590/S1809-98232011000200003.

Maia, M. A. I. A. R., \& Torres, H. de C. (2016). Associação do tempo de contato no programa educativo em diabetes mellitus no conhecimento e habilidades de autocuidado de autocuidado. São Paulo, SP: Revista da Escola de Enfermagem da USP, 50(1), 59-65. Recuperado em 18 novembro, 2016, de: http://dx.doi.org/10.1590/S0080623420160000100008 .

Maibach, E., \& Murphy, D. A. (1995). Self-efficacy in health promotion research and practice: conceptualization and measurement. Health Education Research, 10(1), 37-50. Recuperado em 12 novembro, 2016, de: https://doi.org/10.1093/her/10.1.37.

Maseda, A., Millán-Calenti, J. C., Lorenzo-López, L., \& Núñez-Naveira, L. (2013). Efficacy of a computerized cognitive training application for older adults with and without memory impairments. Aging Clinical and Experimental Research, 25(1), 411-419. Recuperado em 11 novembro, 2016, de: https://www.ncbi.nlm.nih.gov/pubmed/23780693.

Michaelis. (2000). Moderno Dicionário da Língua Portuguesa. São Paulo, SP: Melhoramentos.

Morais, O. N. P. de (2009). Grupos de Idosos: Atuação da Psicogerontologia no Enfoque Preventivo. Brasília, DF: Psicologia Ciência e Profissão, 29(4), 846-855. Recuperado em 15 novembro, 2016, de: http://pepsic.bvsalud.org/scielo.php?script=sci_arttext\&pid=S141498932009000400014.

Neri, A. L. (2008). Palavras-chave em Gerontologia (3ª ed.). São Paulo, SP: Alínea.

Nicolato, F. V., Couto, A. M. do, \& Castro, E. A. B. de. (2016). Capacidade de autocuidado de idosos atendidos pela consulta de enfermagem na atenção secundária à saúde. Revista de Enfermagem do Centro Oeste Mineiro, 6 (2), 2199-2221. Recuperado em 12 novembro, 2016, de: http://dx.doi.org/10.19175/recom.v6i2.1016.

Ogden, J. (2004). Psicologia da Saúde. Lisboa, Portugal: Climepsi Editores.

Papalia, D. E., Olds, S. W., \& Feldman, R. D. (2006). Desenvolvimento Humano. D. Bueno, Trad. Porto Alegre, RS: Artmed. (Obra original publicada em 2001).

Pereira, D. A., Costa, N. M. da S. C., Sousa, A. L. L., Jardim P. C. B. V., \& Zanini. C. R. de O. (2012). Efeito de intervenção educativa sobre o conhecimento da doença em pacientes com diabetes mellitus. Ribeirão Preto, SP: Revista Latino-Americana de Enfermagem, 20(30), 478-485. Recuperado em 12 novembro, 2016, de: http://dx.doi.org/10.1590/S010411692012000300008 . 
Ribeiro, P. C. C., \& Yassuda, M. S. (2007). Cognição, estilo de vida e qualidade de vida na velhice. In: Neri, A. L. (Org.). Qualidade de vida na velhice: enfoque multidisciplinar, 189204. Campinas, SP: Atheneu.

Rodin, J., \& Langer, E. J. (1996). Long-term effects of a control-relevant intervention with the institutionalized aged. In: Fein, S., \& Spencer, S. Readings in Social Psychology: The Art and Science of Research, 175-180. Boston: MA: Houghton Mifflin.

Rodríguez-Gázquez, M. de los Á, Arredondo-Holguín, E., \& Herrera-Cortés, R. (2012). Efetividade de um programa educativo em enfermagem no autocuidado em pacientes com insuficiência cardíaca: ensaio clínico randomizado. Revista Latino-Americana de Enfermagem, 20(2), 1-11. Recuperado em 13 novembro, 2016, de: http://dx.doi.org/10.1590/S0104-11692012000200012.

Rojas, M. D. C., \& Villanea, M. S. (2014). Rendimiento cognitivo de personas mayores que participan en grupos organizados en la provincia de San Jose. Actualidades en Psicología, 28(116), 41-53. Recuperado em 08 novembro, 2016, de: http://pepsic.bvsalud.org/scielo.php?script=sci_abstract\&pid=S0258-64442014000100005.

Sandberg, P., Rönnlund, M., Nyberg, L., \& Neely, S. A. (2014). Executive process training in young and old adults. Aging, Neuropsychology, and Cognition, 21(5), 577-605. Recuperado em 12 novembro, 2016, de: http://dx.doi.org/10.1080/13825585.2013.839777.

Santos, V. dos. (1989). Proposta alternativa de ensino-aprendizagem para o autocuidado, uma contribuição da enfermagem, ao enfrentamento da dor e equalização do estresse. Tese de doutorado Universidade de São Paulo, Escola de Enfermagem de Ribeirão Preto, Programa de Pós-graduação em Enfermagem, Ribeiräo Preto, SP.

Sato, A. T., Batista, M. P. P., \& Almeida, M. H. M. de. (2014). Programas de estimulação da memória e funções cognitivas relacionadas. São Paulo, SP: Revista de Terapia Ocupacional da Universidade de São Paulo, 25(1), 51-59. Recuperado em 15 novembro, 2016, de: http://dx.doi.org/10.11606/issn.2238-6149.v25i1p51-59.

Schwarzer, R., \& Fuchs, R. (1995). Self-efficacy and health behaviors. In: Connor, M., \& Norman, P. (Eds.). Predicting Health Behaviour, 163-196. Buckingham: UK: Open University Press.

Shields, C. A., Brawley, L. R. (2006). Preferring proxy-agency: impact on self-efficacy for exercise. Journal Health Psychology, 11(6), 904-914. Recuperado em 13 novembro, 2016, de: https://www.ncbi.nlm.nih.gov/pubmed/17035262.

Silva, B. M. C. da. (2012). Terapia comportamental no controle da incontinência urinária da mulher idosa: uma ação de enfermagem na promoção do autocuidado. Dissertação de mestrado, Universidade do Estado do Rio de Janeiro, Programa de Pós-Graduação em Enfermagem, Rio de Janeiro, RJ. Recuperada em 13 novembro, 2016, de: http://bdtd.ibict.br/vufind/Record/UERJ_bc02a5a723e6120a2438ecf9c6a687b6.

Silva, L. dos S. V., Silva, T. B. L. da, Falcão, D. V. da S., Batistoni, S. S. T., Lopes, A., Cachioni, M., Neri, A. L., \& Yassuda, M. S. (2014). Relações entre queixas de memória, sintomas depressivos e desempenho cognitivo em idosos residentes na comunidade. São Paulo, SP: Revista Psiquiatria Clínica, 41(3), 67-71. Recuperado em 12 novembro, 2016, de: http://www.periodicos.usp.br/acp/article/viewFile/84283/87130.

Silva, M. J. da. (2000). O idoso submetido à cirurgia de próstata: intervenção do enfermeiro para o autocuidado. Dissertação de mestrado, Universidade do Estado do Rio de Janeiro Programa de Pós-Graduação em Enfermagem, Rio de Janeiro, RJ. 
Silva, S. G. M. R. N. (2009). Qualidade de Vida e Bem-Estar Psicológico em Idosos. Dissertação de mestrado, Universidade Fernando Pessoa, Programa de Pós-Graduação em Psicologia Clínica e da Saúde, Porto, Portugal.

Silva, T. B. L., Oliveira. A. C. V. de. Paulo, D. L. V., Malagutti, M. P., Danzini, V. M., Danzini, \& Yassuda, M. S. (2011). Treino cognitivo para idosos baseado em estratégias de categorização e cálculos semelhantes a tarefas do cotidiano. Rio de Janeiro, RJ: Revista Brasileira de Geriatria e Gerontologia, 14(1), 65-74. Recuperado em 09 novembro, 2016, de: http://dx.doi.org/10.1590/S1809-98232011000100008.

Torres, H. de C., Franco, L. J., Stradioto, M. A., Hortale, V. A., \& Schall, V. T. (2009). Avaliação estratégica de educação em grupo e individual no programa educativo em diabetes. Rio de Janeiro, RJ: Revista de saúde pública, 43(2), 291-298. Recuperado em 07 novembro, 2016, de: http://dx.doi.org/10.1590/S0034-89102009005000001.

Verdan, C., Casarsa, D., Perrout, M. R., Santos, M., Souza, J. A. de, Nascimento, O., Coutinho, E. S. F., \& Laks, J. (2014). Lower mortality rate in people with dementia is associated with better cognitive and functional performance in an outpatient cohort. São Paulo, SP: Arquivos de Neuropsiquiatria, 72(4), 278-282. Recuperado em 12 novembro, 201, de: http://dx.doi.org/10.1590/0004-282X20140003.

Yassuda, M. S., \& Abreu, V. P. S. (2006). Avaliação cognitiva. In: Freitas, E. V., Py, L., Cançado, F. A. X., Doll, J., \& Gorzoni, M. L. (Eds.). Tratado de geriatria e gerontologia, 1252-1266. Rio de Janeiro, RJ: Koogan.

Recebido em 01/05/2017

Aceito em 30/06/2017

Eunice Maria Godinho Morando - Professora aposentada da UFJF. Psicóloga. Membro do Laboratório de Estudos do Corpo LABESC/UFJF. Doutoranda do Programa de PósGraduação em Psicologia. Mestre em Psicologia da Educação, IESAE/Fundação Getúlio Vargas, RJ. Especialista em Educação Brasileira, CES/JF. Especialista em Psicologia Gestalt e Transpessoal, Associação Brasileira de Psicologia. Especialista em Gestal-Terapia, Instituto Gestalt do Rio de Janeiro.

E-mail: e.morando@hotmail.com 
Juliana Campos Schmitt - Mestre pelo Programa de Pós-Graduação em Psicologia da Universidade Federal de Juiz de Fora. Graduada em Letras e graduanda em Pedagogia, UFJF. Integrante do grupo CogLin, Cognição e Linguagem, Psicologia - UFJF.

E-mail: julianaschmittufjf@hotmail.com

Maria Elisa Caputo Ferreira - Licenciada em Educação Fisica, Universidade Federal de Juiz de Fora. Bacharel em Serviço Social, Faculdade de Serviço Social do Rio de Janeiro. Especialista em Docência Universitária, Universidade Castelo Branco. Mestrado em Educação Física, Universidade Gama Filho. Mestrado em Ciência da Motricidade Humana, pela Universidade Castelo Branco. Doutorado e Pós-doutorado em Educação, Universidade de São Paulo. Atualmente, é professora adjunta, Faculdade de Educação Física e Desportos, FAEFID, Universidade Federal de Juiz de Fora, UFJF. Líder do Grupo de Pesquisa Corpo e Diversidade, do Conselho Nacional de Desenvolvimento Científico e Tecnológico (CNPq). Orientadora Plena nos programas de Pós-Graduação Associado em Educação Física UFVUFJF e em Psicologia ICH-UFJF.

E-mail: caputoferreira@terra.com.br 